\title{
CONTRIBUCIÓN AL CONOCIMIENTO DE LOS HONGOS HYPHOMYCETES DE MÉXICO
}

\author{
Gabriela Heredia \\ Rosa María Arias \\ Y \\ Manuela Reyes E. \\ Instituto de Ecología, A.C. \\ Apartado postal 63 \\ 91000 Xalapa, Veracruz
}

\begin{abstract}
RESUMEN
Se registran por primera vez para México y se describen 10 especies de Hyphomycetes asociadas a restos vegetales, colectadas en diversas zonas tropicales de México. Las descripciones se basan en las características morfológicas de los especímenes in situ. Algunas especies fueron aisladas en medio de cultivo. Se anexa información sobre la distribución y características distintivas de cada hongo. La mayoría de las especies estudiadas son poco conocidas a nivel mundial.
\end{abstract}

\begin{abstract}
Ten dematiaceous species collected in plant debris, are recorded for the first time in several tropical and semitropical localities of Mexico. Descriptions are based on their morphology in situ. Some species were isolated into culture. Additional information on their geographical distribution and taxonomic characteristics are presented. Most of the species studied have been scarcely recorded throughout the world.

\section{INTRODUCCIÓN}

Los hongos forman un grupo sumamente abundante y morfológicamente diverso. Algunas especies pueden subsistir como parásitas y otras como saprobias asociadas a restos vegetales. En particular, estas últimas han sido poco estudiadas en México. Aunque en la última década se han publicado algunas contribuciones de carácter taxonómico (Heredia, 1994; Heredia et al., 1997, Heredia y Mercado-Sierra, 1998), en las que se incluyen registros para Veracruz, Tamaulipas y Campeche, la magnitud de la diversidad de estos hongos a nivel nacional está muy lejos de conocerse. El presente trabajo es una contribución al estudio de los Hyphomycetes de México y tiene como objetivo primordial describir 10 especies hasta ahora desconocidas para la micobiota del país.
\end{abstract}




\section{METODOLOGÍA}

El material estudiado fue colectado en diversas localidades semitropicales y tropicales de los estados de Campeche, Chiapas, Tamaulipas y Veracruz. Las muestras recolectadas consistieron en pedazos de troncos, hojarasca, ramas e inflorescencias. En el laboratorio se prepararon cámaras húmedas para estimular la esporulación. Las características de las especies que a continuación se describen fueron extraídas directamente del material colectado. Para la revisión microscópica se realizaron preparaciones fijas en alcohol polivinílico. Paralelamente se efectuaron observaciones con el microscopio electrónico de barrido, para lo cual las muestras fueron sombreadas con oro paladio por 15 minutos, y revisadas en un microscopio JEOL Mad JSM-T20.

Dependiendo de la disposición de material, se intentó aislar algunas especies en los siguientes medios de cultivo: papa dextrosa agar (PDA), harina de maíz agar (HMA) y extracto de malta agar (EMA). Los ejemplares de referencia están depositados en el herbario del Instituto de Ecología, A.C. (XAL).

\section{DESCRIPCIÓN DE LAS ESPECIES}

Acrodictys stilboidea J. Mena et Mercado, Acta Botanica Hungarica 32: 190. 1986. Figs. 1a$1 \mathrm{~b}$.

Colonias sobre el sustrato dispersas, pilosas. Micelio mayormente inmerso en el sustrato. Sinemas claviformes, hasta de $300 \mu \mathrm{m}$ de largo por 21-32 $\mu \mathrm{m}$ en la base. Conidióforos macronematosos, sinematosos, simples, rectos, lisos, septados, unidos hasta cerca de la zona apical en donde se separan en forma penicilada, negros o cafés oscuros en conjunto y oliváceos cuando están separados, de 1.8-3 $\mu \mathrm{m}$ de grosor en la parte superior. Células conidiógenas monoblásticas, integradas, terminales, percurrentes, cilíndricas, subhialinas a oliváceas. Conidios solitarios, acrógenos, lisos, muriformes, elipsoidales o piriformes, café oliváceos, de (22-)30-43 x (14)17-23 $\mu \mathrm{m}$, con una célula basal protuberante, obcónica, subhialina, de 5-7 $\mu \mathrm{m}$ de grosor en su parte más ancha, de 3-5 $\mu \mathrm{m}$ en la base truncada.

Material examinado y sustrato: VERACRUZ. Cosautlán. CB631-4, 18/II/96. VERACRUZ. Rancho Guadalupe, Xalapa. CB720, 11/I/99. Cerro Macuiltépetl, Xalapa. CB721, 5/VII/98. Sobre ramas leñosas desprendidas.

Observaciones: Las características morfológicas, el tipo de conidiogénesis holoblástica y la secesión esquizolítica de los conidios, ubican sin duda a nuestro ejemplar como $A$. stilboidea. El material examinado amplía el intervalo conocido de las dimensiones de la especie, puesto que la longitud máxima de los sinemas del material estudiado (300 $\mu \mathrm{m})$ es menor que la del tipo $(575 \mu \mathrm{m})$ y por el contrario los conidios son más grandes (22$43 \times 14-23 \mu \mathrm{m})$ que los descritos $(22-31 \times 13-16.5 \mu \mathrm{m})$ con anterioridad.

Acrodictys stilboidea es una especie que se distingue del resto de los Acrodictys descritos por la agrupación de conidióforos en sinemas (Mercado-Sierra y Mena-Portales, 
Heredia et al.: Contribución al Conocimiento de los Hyphomycetes de México

1986). Es interesante mencionar que Acrodictys stilboidea tiene una estrecha similitud morfológica con el género Kostermansinda Rifai, dentro del cual se han descrito las especies K. magna (Boedijn) Rifai y K. minima Cabello et Aram. (Ellis, 1971a; Arambarri et al., 1987). Khutubutheen y Nawawi (1994) indican que en el género Kostermansinda la secesión conidial se efectúa rexolíticamente quedando restos de la pared celular, tanto en la célula conidiógena como en los conidos. Dicha característica es difícil de apreciar en las ilustraciones del material tipo de K. minima, especie que es muy semejante a $A$. stilboidea en tamaño.

Desde su descripción sobre ramas muertas de Zanthoxylum sp. en Cuba (MercadoSierra y Mena-Portales, 1986), A. stilboidea no había sido registrada de nuevo en la literatura.

Atractilinia biseptata R. F. Castañeda, Fungi Cubense. Págs. 2-3. 1986. Fig. 2.

Colonias sobre el sustrato efusas, pilosas, distribuidas a lo largo de la vena principal de las hojas. Conidióforos agregados en sinemas o fascículos, compactados en la base y expandidos en la parte apical, simples, lisos, septados, cafés oscuros en la base, aclarándose hacia el ápice, de hasta $450 \mu \mathrm{m}$ de alto x 17-40 $\mu \mathrm{m}$ de ancho en la base del fascículo. Células conidiógenas poliblásticas, integradas, terminales e intercalares, simpodiales, denticuladas, de color café claro a hialinas, de 15-45 $\mu \mathrm{m}$ de alto $\times 4 \mu \mathrm{m}$ de ancho. Conidios acropleurógenos, fusiformes, biseptados, ligeramente rugosos, de hialinos a subhialinos, con la célula basal truncada y cicatrizada, la célula distal más alargada y subulada, de la base a la punta los conidios miden 30-37 $\mu \mathrm{m}$ de largo x 4-7 $\mu \mathrm{m}$ de ancho.

Material examinado y sustrato: CHIAPAS. Reserva de la Biosfera "Montes Azules". CB722, 23/VI/99. Sobre hojas muertas.

Características en cultivo. Esta especie fue aislada en PDA y HMA. En PDA las colonias alcanzaron $30 \mathrm{~mm}$ de diámetro en un mes. Las colonias jóvenes presentaron tonalidades blanquecinas y posteriormente se volvieron verde grisáceas, quedando la mayor parte del micelio inmerso en el medio. A la semana de la inoculación se formaron conidióforos solitarios con conidios. No se observó la formación de sinemas.

En HMA, el hongo generó escaso micelio, inmerso en el sustrato con tonalidades pardas claras. A los cinco días se observaron conidióforos fértiles en fascículos dispersos en el medio y cubiertos por exudados hialinos en la punta.

Observaciones: Las características morfológicas y las dimensiones del material revisado coinciden con la descripción de Castañeda-Ruíz (1986) exceptuando el ancho de los conidios que en nuestro caso es mayor (4-7 $\mu \mathrm{m}$ vs. 3-4 $\mu \mathrm{m}$ en el material tipo).

Desde que fue descrita en Cuba (Castañeda-Ruíz, 1986) sobre pecíolos no identificados, esta especie no se había vuelto a registrar sino hasta el presente trabajo. 
Beltraniella clavatophora R. F. Castañeda, Cano et Guarro, Mycotaxon 58: 246-248. 1996. Fig. 3.

Colonias sobre el sustrato puntiformes, distribuidas a lo largo de la vena principal de la hoja. Conidióforos macronematosos, mononematosos, erectos, rectos, generalmente simples, rara vez ramificados, lisos, septados, claviformes en el ápice, de color café claro, subhialinos hacia la parte superior, hasta de $228 \mu \mathrm{m}$ de largo x 5-6 $\mu \mathrm{m}$ de ancho arriba de la base y 4.5-5 $\mu \mathrm{m}$ en el ápice. Células conidiógenas poliblásticas, simpodiales, denticuladas, hialinas, lisas, de 7-10 $\mu \mathrm{m}$ de largo x 5-7 $\mu \mathrm{m}$ de ancho. Células intermedias generalmente ausentes, obovoides, limoniformes, lisas, hialinas, 7-8 x 4-5 $\mu \mathrm{m}$. Conidios turbinados, subtruncados en el ápice, adelgazándose hacia la base, unicelulares, en la región superior con una banda hialina transversa y ligeramente constreñida; de 19-25 $\mu \mathrm{m}$ de largo x 5-6 $\mu \mathrm{m}$ de ancho.

Material examinado y sustrato: CAMPECHE. Reserva de la Biosfera "Calakmul". CB722, 10/XI/98. TAMAULIPAS. Tampico Alto. CB724, 27/III/98. Sobre hojas muertas no identificadas.

Observaciones: El presente registro representa el primero después de la descripción de la especie. El material tipo fue colectado en Cuba sobre hojas muertas de Manilkara zapota (Castañeda-Ruíz et al., 1996).

Candelabrum brocchiatum Tubaki, Trans. Mycol. Soc. Japan 16: 134-137. 1975. Fig. 5.

Colonias sobre el sustrato puntiformes, distribuidas a lo largo de la superficie vegetal. Conidióforos macronematosos, mononematosos, lisos, hialinos, de hasta $45 \mu \mathrm{m}$ de largo x 1.5-2 $\mu \mathrm{m}$ de ancho. Células conidiógenas monoblásticas, terminales, determinadas, ligeramente infladas. Conidios solitarios, acrógenos, esféricos, de subhialinos a anaranjados pálidos al madurar, ramificados varias veces dicotómicamente con prominentes dentículos en las puntas; de 61-130 $\mu \mathrm{m}$ de diámetro.

Material examinado y sustrato: VERACRUZ. Rancho Guadalupe., Xalapa. CB725, 15/ IV/98. VERACRUZ. Cerro Macuiltépetl, Xalapa. CB726, 5/VII/98. Sobre ramas sumergidas.

Observaciones: El género Candelabrum van Beverw. incluye hongos aereoacuáticos, generalmente asociados a restos vegetales sumergidos. A la fecha se han descrito seis especies, $C$. brocchiatum se distingue principalmente por el tamaño de sus conidios, los cuales son más grandes que en las otras especies. C. brocchiatum ha sido colectado en madera sumergida y hojas en Japón, Nueva Zelanda (Tubaki, 1975) y Australia (Hyde y Goh, 1998). 
Heredia et al.: Contribución al Conocimiento de los Hyphomycetes de México

Endocalyx melanoxanthus (Berk. et Br.) Petch, Ann. Bot. Lon. 22: 390. 1908. Figs. 6a y $6 b$.

Colonias sobre el sustrato compuestas por una trama micelial superficial, cupulada, amarilla brillante con masas negras de conidios en el centro, de hasta $650 \mu \mathrm{m}$ de diámetro. Conidióforos basáuxicos, macronematosos, mononematosos, cafés claros, lisos, de 1-2 $\mu$ m de diámetro. Células conidiógenas integradas, terminales e intercalares, cilíndricas, denticuladas. Conidios acropleurógenos, simples, lenticulares, aplanados, negros, lisos, con una hendidura germinativa más clara en la parte media, de 11-15 x 9-12 $\mu \mathrm{m}$ de frente y 7-9 $\mu \mathrm{m}$ de grosor.

Material examinado y sustrato: VERACRUZ. Centro de Investigaciones Costeras "La Mancha” (CICOLMA) km 37.5 carretera Cardel-Nautla. CB095-1; CB096-4, 10/XI/93. Sobre raquis de Acrocomia mexicana. VERACRUZ. Ibid. CB319-1, 4/VI/94. Sobre un tronco de Arecaceae.

Esta especie es común en palmas, ha sido registrada en muy diversas zonas tropicales de África (Hughes, 1952), Asia (Ellis, 1971a; Matsushima, 1980) y Australia (Hughes, 1978). En América hasta antes del presente trabajo se conocía para Estados Unidos (Florida), (Sutton, 1978), Cuba (Mercado-Sierra, 1982) y Perú (Matsushima, 1993). Seguramente su distribución es pantropical.

Paraulocladium fabisporum Kuthub. et Nawawi, Mycol. Res. 98: 679. 1994. Fig. 11.

Colonias sobre el sustrato efusas, pilosas, pardas oscuras. Conidióforos macronematosos, mononematosos, erectos, rectos o flexuosos, geniculados en la parte superior, simples, septados, lisos, cafés oscuros en la base, aclarándose ligeramente hacia el ápice, de hasta $175 \mu \mathrm{m}$ de alto $\times 3 \mu \mathrm{m}$ de ancho en la base. Células conidiógenas politréticas, integradas, terminales, simpodiales, geniculadas, cicatrizadas, cafés a cafés claras, hasta de $80 \mu \mathrm{m}$ de largo x 3-4 $\mu \mathrm{m}$ de ancho. Conidios acropleurógenos, simples, reniformes, redondeadas en los extremos, aplanados, lisos, con una pequeña cicatriz marcando el área de adhesión a la célula conidiógena, constreñidos a lo largo de un septo longitudinal y 2 distoseptos transversales, café dorados, de 11-13 $\mu \mathrm{m}$ de largo x 8-9 $\mu \mathrm{m}$ de ancho.

Material examinado y sustrato: VERACRUZ. Nanciyaga. CB727, 1/VI/98. Sobre troncos muertos.

Observaciones: El género Paraulocladium incluye las especies $P$. angulisporum $\mathrm{R}$. $F$. Castañeda y $P$. fabisporum, las cuales se diferencian por el tamaño y forma de los conidios. El presente registro es el primero desde que la especie fue descrita de ramas muertas colectadas en Malasia (Kuthubutheen y Nawawi, 1994) y representa el primero para la micobiota neotropical. 
Pseudopetrakia kambakkamensis (Subram.) M. B. Ellis, Mycol. Pap. 125: 4. 1971. Figs. 7 y 12.

Colonias sobre el sustrato efusas, granulares, irregulares, café oscuro negruzcas. Conidióforos micronematosos o semimacronematosos, erectos, simples o ramificados, septados, ligeramente rugosos, cafés oscuros, hasta de $10 \mu \mathrm{m}$ de largo x 4-8 $\mu \mathrm{m}$ de ancho. Células conidiógenas monoblásticas, integradas, terminales, determinadas, cafés oscuras. Conidios solitarios, terminales, elipsoidales, de formas variadas, esféricos, obovoides, algunos ligeramente curvos, muriformes, cafés oscuros, de 22.5-43.8 $\mu \mathrm{m}$ de largo x 18-24 $\mu \mathrm{m}$ de ancho, cubiertos en toda la superficie por prominentes verrugas oscuras y en la parte superior por 1-4 espinas negras de 11-51 $\mu \mathrm{m}$ de largo por 6-10 $\mu \mathrm{m}$ de ancho en la base.

Material examinado y sustrato: VERACRUZ. Centro de Investigaciones Costeras "La Mancha" (CICOLMA) km 37.5 carretera Cardel-Nautla. CB090, 10/XI/93. Sobre troncos muertos.

Observaciones: Pseudopetrakia kambakkamensis es una especie lignícola. Se conoce poco sobre su distribución, el material tipo proviene de madera muerta colectada en Kambakkam, India (Ellis, 1971b). Posteriormente fue registrada para Cuba (HolubováJechová y Mercado-Sierra, 1984; 1989).

Speiropsis hyalospora Subram. et Lodha, Can. J. Bot. 42: 1060-1063. 1964. Fig. 9.

Colonias sobre el sustrato efusas, expandidas en la superficie vegetal, micelio inmerso en el sustrato. Conidióforos macronematosos, mononematosos, erectos, rectos, septados, lisos, con ramificaciones cortas en el ápice, cafés pálidos, de 58-80 $\mu \mathrm{m}$ de largo x 4-5 $\mu \mathrm{m}$ de ancho. Células conidiógenas localizadas en la parte superior del conidióforo, poliblásticas con dentículos en el ápice, surgen en grupos, de forma variable, ensanchadas hacia la punta, hialinas o subhialinas, de 8-9 $\mu \mathrm{m}$ largo por 4-6 $\mu \mathrm{m}$ de ancho en la parte superior. Conidios multicelulares, surgen de los dentículos de las células conidiógenas, compuestos de 3-4 ramas dispuestas en forma de abanico, cada rama formada por células hialinas unidas por un istmo, la rama central presenta de 6 a 8 células, la célula basal puede ser cilíndrica o cónica, las intermedias son cilíndricas y las apicales cónicas, de la base al ápice la rama central mide hasta $75 \mu \mathrm{m}$ de largo x 4-5 $\mu \mathrm{m}$ de ancho, las células individuales miden de 8-10 $\mu \mathrm{m}$ de largo $\times$ 4-5 $\mu \mathrm{m}$ de ancho.

Material examinado y sustrato: VERACRUZ. Estación de Biología Tropical "Los Tuxtlas". CB728, 7/X/98. Sobre hojas muertas.

Características en cultivo. Se efectuaron aislamientos en EMA, PDA y HMA. En todos los casos el hongo formó colonias inmersas en el sustrato con escaso micelio aéreo. En un principio las colonias presentaron tonalidades grisáceas, oscureciéndose posteriormente. El desarrollo de conidióforos y conidios fue observado únicamente en HMA, a los diez días después de la inoculación. 
Heredia et al.: Contribución al Conocimiento de los Hyphomycetes de México

Observaciones: Con excepción del tamaño de los conidióforos, el cual es menor (58$80 \mu \mathrm{m})$ en el material estudiado que en el ejemplar tipo $(80-120 \mu \mathrm{m})$, el resto de los caracteres morfológicos concuerdan con los descritos para Speiropsis hyalospora.

Esta especie ha sido colectada en hojas de Araucaria en Sri Lanka y aislada de estiércol de caballo en la India (Subramanian y Lodha, 1964; Ellis, 1976). El presente registro es el primero para la micobiota neotropical.

Speiropsis scopiformis Kuthub. et Nawawi, Trans. Br. Mycol. Soc. 89: 584-587. 1987. Figs. $10 \mathrm{a}$ y $10 \mathrm{~b}$.

Colonias sobre el sustrato efusas, expandidas en la superficie vegetal, micelio inmerso en el sustrato. Conidióforos macronematosos, mononematosos, solitarios, erectos, rectos, simples, septados, lisos, con consecutivas ramificaciones cortas en el ápice, cafés pálidos, de 57-65 $\mu \mathrm{m}$ de largo x 4-5 $\mu \mathrm{m}$ de ancho. Células conidiógenas poliblásticas, simpodiales, surgen de la parte superior del conidióforo formando cadenas de hasta nueve células, clavadas, denticuladas en el ápice, de cafés pálidas a subhialinas, de 6-9 $\mu \mathrm{m}$ de largo x 3-4 $\mu \mathrm{m}$ de ancho. Conidios compuestos de 5-7 células conectadas por un istmo formando una cadena hialina, las células de los extremos son cónicas y las intermedias cilíndricas, las células del ápice miden de 5.5-8 $\mu \mathrm{m}$ de largo por 1.5-2 $\mu \mathrm{m}$ de ancho, las intermedias miden de 6-10 $\mu \mathrm{m}$ de largo por 2-3 $\mu \mathrm{m}$ de ancho, las cadenas alcanzan hasta $50 \mu \mathrm{m}$ de largo $\times 2-3 \mu \mathrm{m}$ de ancho.

Material examinado y sustrato: VERACRUZ. Cerro Macuiltépetl, Xalapa. CB729, 5/ VII/98. CAMPECHE: Reserva de la Biosfera "Calakmul". CB730, 10/XI/98. Sobre hojas muertas.

Colonias en cultivo: Se aisló en EMA, PDA y HMA; en todos los casos el hongo formó colonias inmersas en el sustrato con poco micelio aéreo, de color café grisáceo al principio, pigmentándose posteriormente de café oscuro. Únicamente en HMA se observaron fructificaciones a los ocho días después de la inoculación.

De las seis especies que han sido descritas dentro del género Speiropsis, tres presentan cadenas conidiales no ramificadas; Speiropsis simplex Matsush., S. belauensis Matsush. y S. scopiformis Kuthub. et Nawawi, esta última se distingue de las otras en que presenta conidióforos sin ramificar.

Speiropsis scopiformis ha sido colectada en hojas muertas. El material tipo proviene de Malasia (Kuthubutheen y Nawawi, 1987) y hasta la fecha se tenían registros solamente para Nueva Caledonia (Mouchacca, 1990) y Cuba (Castañeda-Ruíz et al., 1998).

Tetropileus sphaerophorus (Berk. et Curtis) S. Hughes et Deighton, Mycol. Pap. 78: 2-4. 1960. Figs. 4 y 8.

Colonias sobre el sustrato efusas, conspicuas, dispersas en la superficie vegetal, compuestas de estípites anillados expandidas, micelio inmerso en el sustrato. Estípites erectos o ligeramente curvados, cilíndricos, anillados o articulados, compuestos de hifas 
de 1.5-3 um de grosor, dispuestas paralelamente, formando gruesas columnas compactas, las hifas externas cafés oscuras y las internas más claras. La parte superior del estípite sostiene una estructura terminal (gema) desprendible, después de que la gema cae continúa el crecimiento del estípite mediante la elongación de las células internas, formándose un anillo con las hifas externas, lo que le da una apariencia articulada al estípite que puede tener hasta $418 \mu \mathrm{m}$ de largo x 30-56 $\mu \mathrm{m}$ de ancho en la base y 20-25 $\mu \mathrm{m}$ en el ápice. Bulbilo o gema multicelular, obcónica, solitaria, adherida al estípite por una base truncada y ligeramente cóncava, compuesta de hifas septadas, ramificadas, cafés claras, vista de arriba la gema presenta forma esférica con bordes irregulares, alcanza hasta $117 \mu \mathrm{m}$ de diámetro. En la superficie de la gema se observan áreas circulares subhialinas de $2 \mu \mathrm{m}$ de diámetro.

Material examinado y sustrato: VERACRUZ. Centro de Investigaciones Costeras "La Mancha" (CICOLMA) km 37.5 carretera Cardel-Nautla. CB286, 6/VI/94. VERACRUZ. Estación de Biología Tropical "Los Tuxtlas". CB119-1, 22/XI/93; CB205, 23/XI/93. Sobre ramas muertas.

Observaciones: La peculiar morfología de esta especie permite su fácil identificación. En la descripción presentada se ha empleado la terminología de Deighton (1960). Seifert (1990) y Matsushima (1995) consideran como un sinema la estructura nombrada como estípite, sin embargo, tomando en cuenta que hasta ahora se desconoce si las hifas producen algún tipo de propágulo, resulta más apropiado definirla como un estípite. La función de la gema o bulbilo aún se desconoce, sus características y forma de desprendimiento hacen pensar que se trata de una estructura de propagación e incluso se ha sugerido que las zonas circulares translúcidas en su superficie (Fig. 4) podrían ser puntos de germinación (Dodge, 1946). En una de las colectas realizadas por Matsushima (1995) en Tailandia, el autor señala la presencia de fíbulas en las hifas del estípite, lo cual podría significar que este hongo representa una fase anamórfica de algún basidiomicete.

Al parecer T. sphaerophorus es una especie pantropical, la primer colecta de este hongo data de 1946 (Dodge, 1946) en Florida, posteriormente se ha registrado en Sierra Leona (Deighton, 1960), Java (Seifert, 1990), Tailandia, Malasia y Taiwan (Matsushima,1995). En todos los casos ha sido encontrada sobre hojas y ramas muertas.

\section{COMENTARIOS FINALES}

Las especies Acrodictys stilboidea, Atractilina biseptata, Beltraniella clavatophora y Paraulocladium fabisporum no habían sido mencionadas en la literatura desde que fueron descritas por primera ocasión. Los hongos Candelabrum brochiatum, Paraulocladium fabisporum y Speiropsis scopiformis representan primeros registros para la micobiota neotropical.

\section{AGRADECIMIENTOS}

Parte de este trabajo se efectuó gracias al apoyo de la CONABIO (proyecto P030). Las observaciones al microscopio de barrido se realizaron con la ayuda del Sr. Tiburcio 
Heredia et al.: Contribución al Conocimiento de los Hyphomycetes de México

Laez. Vinicio Sosa, Roger Guevara y José Antonio Ángeles amablemente contribuyeron, proporcionándonos muestras de Campeche, Chiapas y Tamaulipas, respectivamente. Deseamos expresar nuestro agradecimiento al micólogo Rafael Castañeda Ruíz por sus observaciones al manuscrito. Se agradece al Instituto de Ecología, A.C. (proyecto 902-07) todo el apoyo brindado.

\section{LITERATURA CITADA}

Arambarri, A., M. Cabello y A. Mengascini. 1987. New hyphomycetes from Santiago river (Buenos Aires province, Argentina). Mycotaxon 29: 29-35.

Castañeda-Ruíz, R. F. 1986. Fungi Cubense. Instituto de Investigaciones Fundamentales en Agricultura Tropical Alejandro de Humboldt. La Habana, Cuba. 20 pp.

Castañeda-Ruíz, R. F., J. Cano y J. Guarro. 1996. Notes on conidial fungi. VII. Two new species of Beltraniella from Cuba. Mycotaxon 58: 243-251.

Castañeda Ruíz, R. F., J. Guarro, E. Malayo y C. Decock. 1998. Notes on conidial fungi. XVI. A new species of Dendryphiosphaera and some new records from Cuba. Mycotaxon 68: 9-19.

Deighton, F. C. 1960. African fungi. I. Mycol. Pap. 78: 1-43.

Dodge, B. O. 1946. A curious fungus on Opuntia. Bull. Torrey Bot. Club. 73: 219-223.

Ellis, M. B. 1971a. Dematiaceous hyphomycetes. Commonwealth Mycological Institute. Kew, England. $608 \mathrm{pp}$.

Ellis, M. B. 1971b. Dematiaceous hyphomycetes. X. Mycol. Pap. 125: 1-30.

Ellis, M. B. 1976. More dematiaceous hyphomycetes. Commonwealth Mycological Institute. Kew, England. $507 \mathrm{pp}$.

Heredia, G. 1994. Hifomicetes dematiáceos en bosque mesófilo de montaña. Registros nuevos para México. Acta Bot. Mex. 27: 15-32.

Heredia, G., J. Mena-Portales y A. Mercado-Sierra. 1997. Hyphomycetes saprobios tropicales. Nuevos registros de dematiáceos para México. Rev. Mex. Mic. 13: 41-51.

Heredia, G. y A. Mercado-Sierra. 1998. Tropical hyphomycetes of Mexico. III. Some species from the Calakmul biosphere reserve, Campeche. Mycotaxon 68: 137-143.

Holubová-Jechová, V. y A. Mercado-Sierra. 1984. Studies on hyphomycetes from Cuba II. Hyphomycetes from the Isla de la Juventud. Cêská Mykol. 38: 96-120.

Holubová-Jechová, V. y A. Mercado-Sierra. 1989. Hyphomycetes from Loma de la Coca and some localities of La Habana and Matanzas provinces, Cuba. Acta Bot. Cubana 76: 1-15.

Hughes, S. J. 1952. Fungi from the Gold Coast. I. Mycol. Pap. 48: 1-91.

Hughes, S. J. 1978. New Zealand fungi 25. Miscellaneous species. New Zealand J. Bot. 16: 311-370.

Hyde, K. D. y T. K. Goh. 1998. Fungi on submerged wood in lake Barrine, north Queensland, Australia. Mycol. Res. 102: 739-749.

Kuthubutheen, A. J. y A. Nawawi. 1987. A new species of Speiropsis from Malaysia. Trans. Brit. Mycol. Soc. 89: 584-587.

Kuthubutheen, A. J. y A. Nawawi. 1994. Henicospora longissima sp. nov., Obeliospora triappendiculata sp. nov., Paraulocladium fabisporum sp. nov. and other hyphomycetes from Malaysia. Mycol. Pap. 98: 677-685.

Matsushima, T. 1980. Saprophytic microfungi from Taiwan. Hyphomycetes. Matsushima Mycological Memoirs 1. Published by the author. Kobe, Japan. $82 \mathrm{pp}$.

Matsushima, T. 1993. Matsushima Mycological Memoirs 8. Published by the author. Kobe, Japan. 75 pp.

Matsushima, T. 1995. Matsushima Mycological Memoirs 9. Published by the author. Kobe, Japan. $54 \mathrm{pp}$. 
Mercado-Sierra, A.1982. Hifomicetes demaciáceos de Cuba. Acta Bot. Cubana 14: 1-7.

Mercado-Sierra, A. y J. Mena-Portales. 1986. Hifomicetes de Topes de Collantes, Cuba I. Especies holoblásticas. Acta Botanica Hungarica 32: 189-205.

Mouchacca, J. 1990. Champignons de Nouvelle-Calédonie - I. Quelques dématiées intéressantes de litière forestière. Persoonia 14: 151-160.

Subramanian C. V. y B. C. Lodha. 1964. Two interesting hyphomycetes. Can. J. Bot. 42: 1057-1063. Seifert, K. A. 1990. Synnematous hyphomycetes. Mem. N.Y. Bot. Gard. 59: 109-154.

Sutton, B. C. 1978. New and interesting hyphomycetes from Tampa, Florida. Mycologia 70: 784-801.

Tubaki, K. 1975. Notes on the Japanese hyphomycetes VI. Candelabrum and Beverwykella gen. nov. Trans. Mycol. Soc. Japan 16: 132-140. 
Heredia et al.: Contribución al Conocimiento de los Hyphomycetes de México

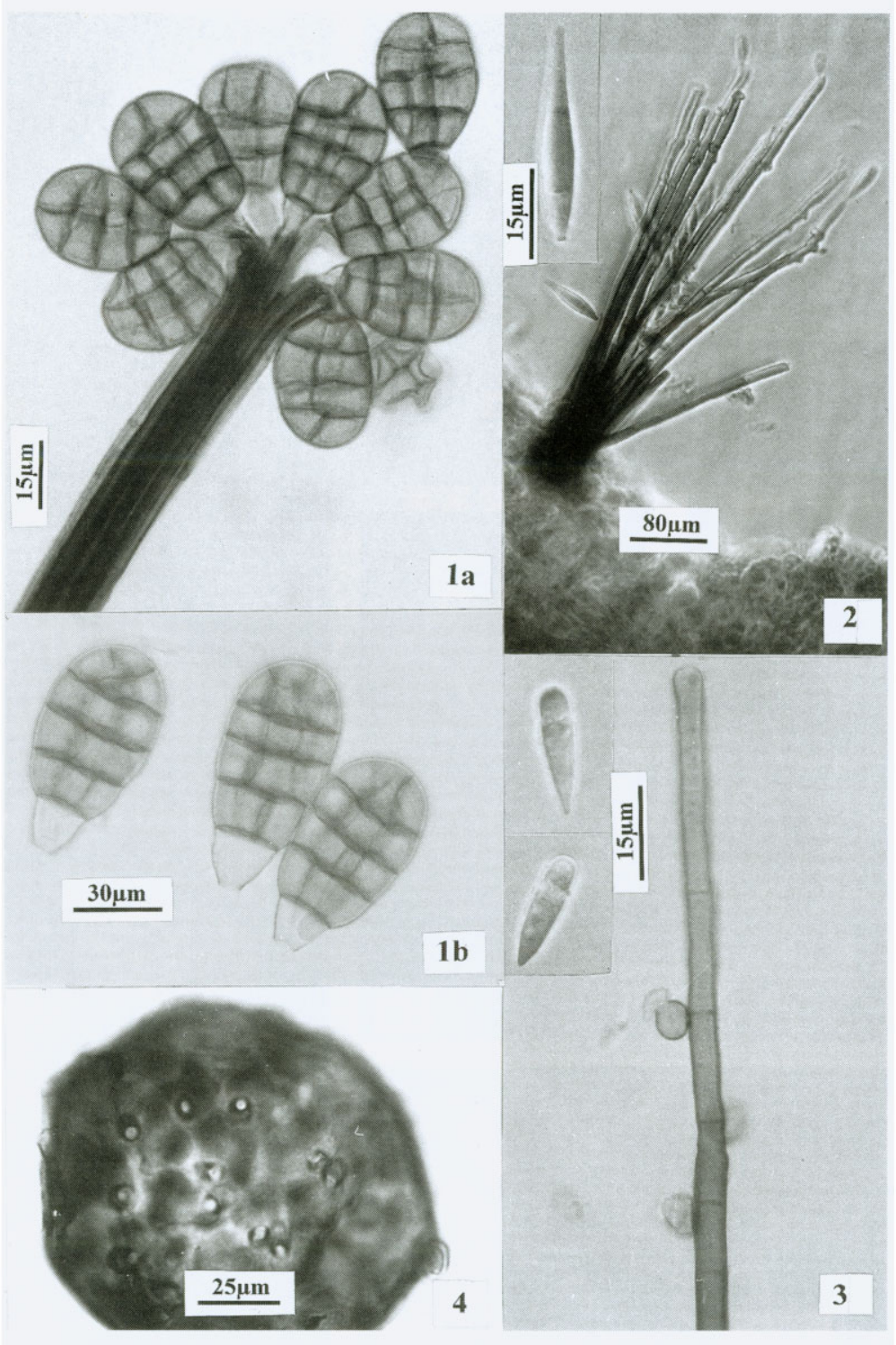

Figs. 1-4. 1a. Acrodictys stilboidea: sinema con conidios. 1b. Acrodictys stilboidea: conidios piriformes con la célula basal hialina y truncada en la base. 2. Atractilinia biseptata: sinema con conidios adheridos; conidio biseptado con la célula basal truncada y cicatrizada y la distal alargada y subulada. 3 . Beltraniella clavatophora: conidióforo claviforme con células conidiógenas denticuladas; conidios turbinados con una banda hialina superior ligeramente constreñida. 4. Tetropileus sphaerophorus: superficie del bulbilo o gema con áreas circulares subhialinas. 

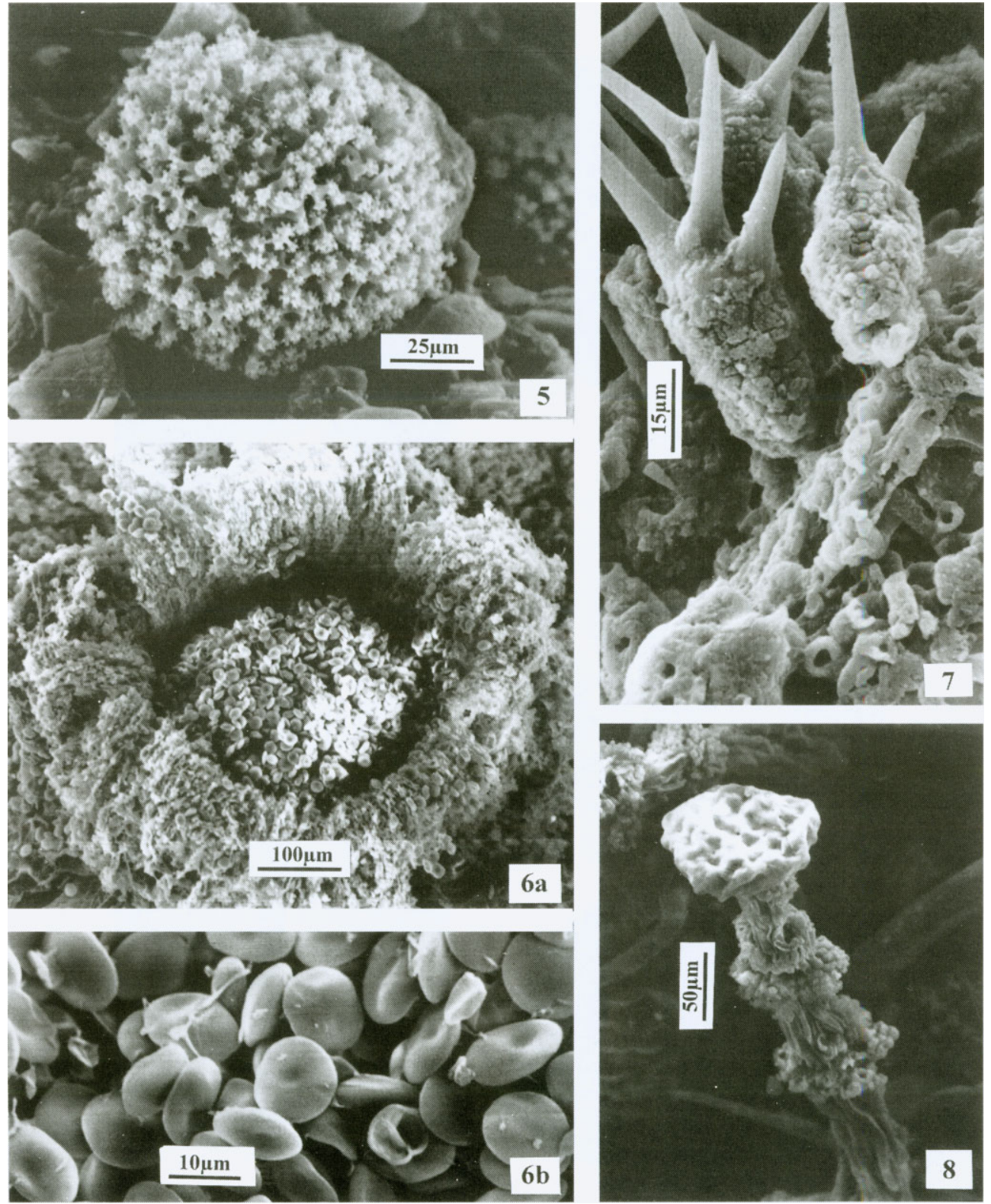

Figs. 5-8. 5. Candelabrum brocchiatum: conidio esférico ramificado dicotómicamente, con dentículos en las puntas. 6a. Endocalyx melanoxanthus: trama micelial cupulada con conidios en la parte central. 6b. Endocalyx melanoxanthus: conidios lenticulares aplanados. 7. Pseudopetrakia kambakkamensis: conidios verrucosos con espinas en la parte superior. 8. Tetropileus sphaerophorus: estípite cilíndrico con anillos o articulaciones y un bulbilo o gema adherido en la parte apical. 
Heredia et al:: Contribución al Conocimiento de los Hyphomycetes de México

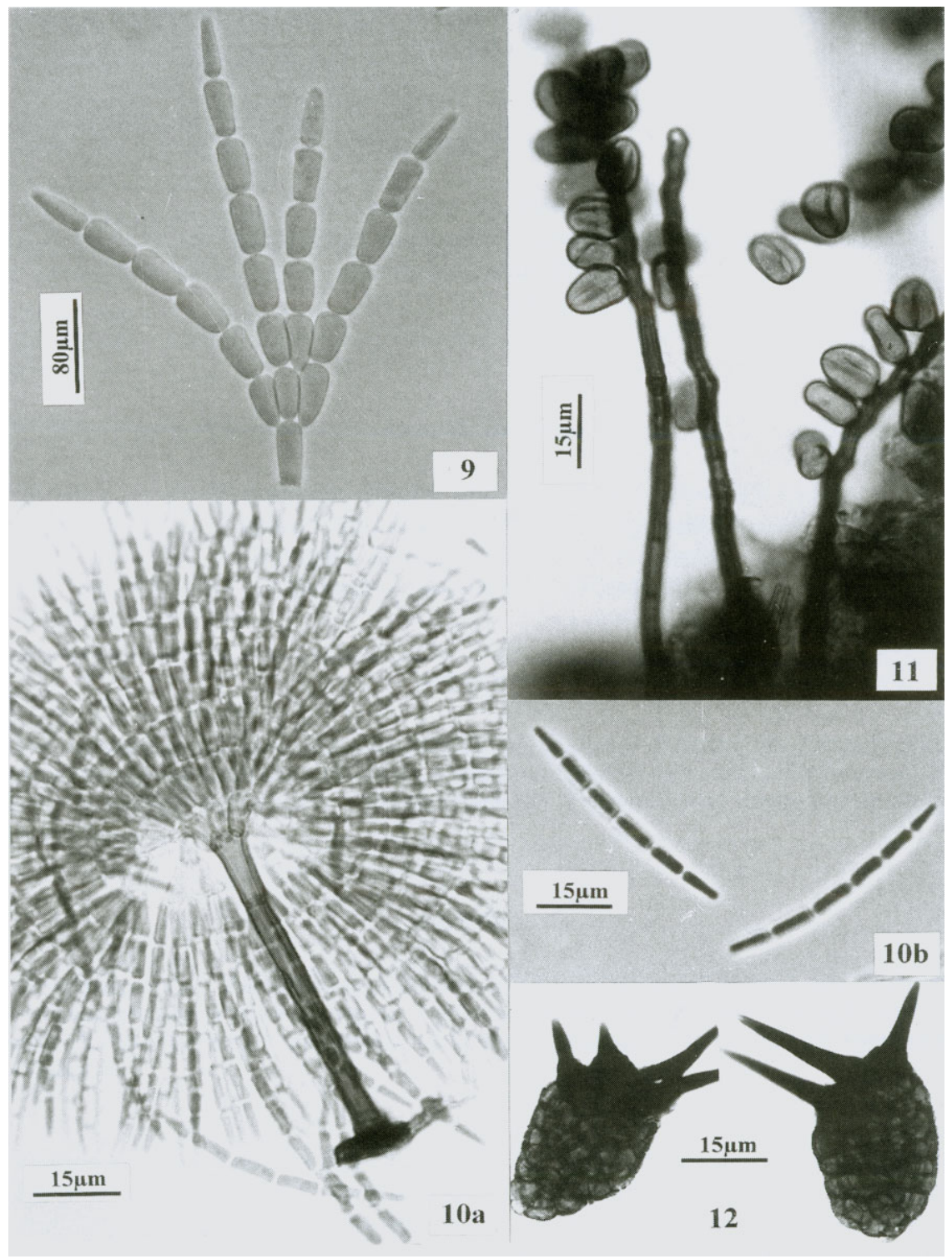

Figs. 9-12. 9. Speiropsis hyalospora: conidio multicelular compuesto de cuatro ramas dispuestas en forma de abanico. 10a. Speiropsis scopiformis: conidióforo con conidios. 10b. Speiropsis scopiformis: conidios multicelulares formando una cadena con celulas distales cónicas. 11. Paraulocladium fabisporum: conidióforo geniculado con conidios reniformes. 12. Pseudopetrakia kambakkamensis: conidios muriformes con verrugas y espinas. 